Revista Brasil. Bot., V.32, n.2, p.405-410, abr.-jun. 2009

Scientific Note / Nota Científica

\title{
Fenologia reprodutiva de Prepusa montana Mart. (Gentianaceae) em uma área de campo rupestre da Chapada Diamantina, BA, Brasil ${ }^{1}$
}

\author{
ALINE GOES COELHO ${ }^{2,3}$ e CAIO GRACO MACHADO ${ }^{2}$
}

(recebido: 07 de fevereiro de 2008; aceito: 20 de março de 2009)

\begin{abstract}
Reproductive phenology of Prepusa montana Mart. (Gentianaceae) in an area of campo rupestre vegetation in the Chapada Diamantina, Bahia State, Brazil). The phenological, flowering and fruiting strategies of Prepusa montana Mart. (Gentianaceae) in an area of campo rupestre vegetation in the Chapada Diamantina were investigated in terms of the influence of local rainfall, relative humidity, temperature, and photoperiod. The numbers of buds, flowers, and mature fruits of this species were monitored during monthly visits to the Mucugê Municipal Park between 6/2006 and 8/2007. Environmental variables (average accumulated rainfall, average temperature and relative humidity) were monitored at the site, while the regional photoperiod was calculated from geographical data. Prepusa montana demonstrated annual flowering of intermediate duration during the dry season. The flowering phenophase did not demonstrate any correlation with rainfall or relative humidity, but was found to be negatively correlated with temperature and photoperiod. Fruit maturation initiated during the dry season and was most intense at the start of the rainy season. Seed dispersal demonstrated a negative correlation with relative humidity. As this species occurs along water courses, flowering appears to be independent of any water stress during the dry period. The negative correlation between fruit opening and relative humidity is associated with the desiccation processes necessary for diaspore maturation and dispersal. The dissemination of $P$. montana seeds is apparently facilitated by the wind during the dry season and then by rainfall at the start of the rainy season (while rainfall would also increase the probability of seedling establishment).
\end{abstract}

Key words - campo rupestre, Chapada Diamantina, Gentianaceae, Prepusa montana, reproductive phenology

RESUMO - (Fenologia reprodutiva de Prepusa montana Mart. (Gentianaceae) em uma área de campo rupestre da Chapada Diamantina, BA, Brasil). Foram investigadas as estratégias fenológicas de floração e frutificação de Prepusa montana Mart. em uma área de campo rupestre da Chapada Diamantina e a sua influência pela pluviosidade, umidade relativa do ar, temperatura e fotoperíodo. Foram registrados os números de flores, botões e frutos maduros desta espécie, em visitas mensais ao Parque Municipal de Mucugê, em Mucugê, BA, de junho de 2006 a agosto de 2007. Os dados das variáveis ambientais (precipitação média acumulada, temperatura média e umidade relativa do ar) foram coletados em Mucugê e o fotoperíodo da área foi calculado por dados geográficos. Prepusa montana apresentou floração anual com duração intermediária, ocorrendo na época seca. A fenofase de floração não apresentou correlação com a pluviosidade e com a umidade relativa do ar, mas apresentou correlação negativa com a temperatura e com o fotoperíodo. A maturação dos frutos iniciou-se na estação seca e teve sua maior intensidade no início da estação chuvosa. A dispersão das sementes apresentou correlação negativa com a umidade relativa do ar. Por ocorrer ao longo de cursos d'água, a floração de $P$. montana parece ser independente do estresse hídrico da época de estiagem. A correlação negativa entre a abertura dos frutos e a umidade relativa do ar está associada ao processo de dessecação, necessário para a maturação e a dispersão dos diásporos. Na estação seca, a disseminação das sementes de $P$. montana pode ser facilitada pelo vento e as chuvas, no início da estação chuvosa, podem também auxiliar na dispersão das sementes, além de garantir maior probabilidade de sua germinação e do estabelecimento das plântulas.

Palavras-chave - campo rupestre, Chapada Diamantina, fenologia reprodutiva, Gentianaceae, Prepusa montana

\section{Introdução}

A família Gentianaceae Juss. apresenta distribuição cosmopolita, com muitas espécies ocorrendo nas regiões

1. Parte da dissertação de mestrado da primeira autora, Programa de Pós-Graduação em Botânica, Universidade Estadual de Feira de Santana, BA, Brasil.

2. Universidade Estadual de Feira de Santana, Departamento de Ciências Biológicas, Avenida Transnordestina, s/nº , Bairro Novo Horizonte, 44036-9000 Feira de Santana, BA, Brasil.

3. Autor para correspondência: alinegcoelho@yahoo.com.br temperadas e frias e em áreas montanhosas intertropicais (Villarreal 1998, Wolff 2005). Segundo Struwe et al. (2002) esta família tem origem tropical, uma vez que a maioria dos clados apresentam grupos tropicais ocupando posições filogenéticas basais, tendo posteriormente se dispersado para as regiões temperadas (Albert \& Struwe 2002).

A América do Sul apresenta o maior número de gêneros desta família e a maior ocorrência de gêneros endêmicos, onde grande parte deles, principalmente os da Tribo Chironieae e Helieae, estão restritos às áreas 
de altitude mais elevadas da Guiana (Tepui) e Brasil (Albert \& Struwe 2002, Struwe et al. 2002). Devido à grande similaridade dos tepuis com os campos rupestres, algumas famílias, incluindo Gentianaceae, cujo centro de diversidade ocorre naquela região, têm na Cadeia do Espinhaço o seu segundo centro de diversidade (Giulietti et al. 1997). No sudeste brasileiro, principalmente nas áreas mais elevadas (campos rupestres e campos de altitude), também ocorre uma alta taxa de endemismo nesta família (Struwe et al. 2002).

Poucos estudos abordam os aspectos fenológicos da família Gentianaceae (Spira \& Pollak 1986, Lennartsson 1997, Wagner \& Mitterhofer 1998, Petanidou et al. 1998, Lennartsson et al. 2000, Petanidou et al. 2001) e nenhum deles se refere às espécies brasileiras.

O estudo da fenologia reprodutiva é um elemento importante na determinação das estratégias evolutivas das diferentes espécies, agregando informações sobre suas relações com fatores abióticos (climáticos) e bióticos (agentes polinizadores e dispersores de sementes) (Morellato \& Leitão-Filho 1992).

Neste contexto, este estudo investigou quais são as estratégias fenológicas de floração e frutificação de Prepusa montana Mart. (Gentianaceae) em uma área de campo rupestre da Chapada Diamantina, Bahia e qual sua relação com algumas variáveis ambientais precipitação média acumulada, fotoperíodo, temperatura e umidade relativa do ar.

\section{Material e métodos}

Área de estudo - A Chapada Diamantina, localizada na porção baiana da Cadeia do Espinhaço, é constituída por um mosaico de tipos vegetacionais, sendo os campos rupestres bastante frequientes. Estes apresentam vegetação herbáceo-arbustiva, que cresce sobre rochas (Giulietti et al. 1997, Harley 1995). Neste ambiente, a variação na topografia, da natureza do substrato e do microclima propiciam a evolução de uma variedade de espécies vegetais endêmicas (Giulietti et al. 1997).

Este estudo foi realizado no Parque Municipal de Mucugê (PMM), localizado no Município de Mucugê, na Chapada

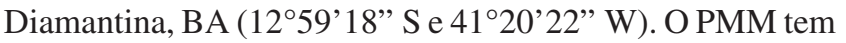
uma área de 450 ha e situa-se, entre cotas de 900 e $1.000 \mathrm{~m}$ de altitude, na Serra do Sincorá, sendo banhado pelos Rios Piabinha, Cumbuca e Mucugê.

O clima na região é caracterizado como úmido a subúmido e semi-árido, com estação chuvosa de outubro a março e estação seca de abril a setembro; a temperatura média anual é de $19{ }^{\circ} \mathrm{C}$, com média mínima de $13{ }^{\circ} \mathrm{C}$, na estação seca, e média máxima de $30^{\circ} \mathrm{C}$, na estação chuvosa (Stradmann 1998).
No PMM predomina a vegetação de campo rupestre, caracterizada por uma fisionomia herbáceo-arbustiva associada a afloramentos rochosos (Conceição \& Giulietti 2002), com grande riqueza de espécies vegetais, decorrente da diversidade de micro-hábitats (Giulietti et al. 1997, Conceição \& Giulietti 2002, Conceição \& Pirani 2005). As famílias mais comuns neste ambiente são Velloziaceae, Orquidaceae, Bromeliaceae, Eriocaulaceae e Asteraceae (Harley 1995).

Espécie estudada - Prepusa montana é uma espécie de hábito arbustivo com, em média, 2,5 m de altura. Suas flores têm características da síndrome quiropterofilia (Faegri \& van der Pijl 1979), porém são visitadas por um grande número de grupos de animais (como beija-flores e abelhas) (observação pessoal, Machado et al. 2007). Seus frutos são do tipo cápsula, deiscentes, e se abrem por fendas longitudinais. As sementes são diminutas, numerosas e angulosas, não apresentando estruturas, como plumas e alas, que facilitem a dispersão a longas distâncias (van der Pijl 1982).

Apesar de não existir estudos sobre a distribuição de P. montana, esta espécie pode ser considerada endêmica da Chapada Diamantina, baseado em dados disponíveis no Herbário do Jardim Botânico do Rio de Janeiro (RB), Herbário da Universidade Estadual de Feira de Santana (HUEFS), Herbário da Universidade de São Paulo (SPF), Missouri Botanical Garden (MO) e New York Botanical Garden (NY), ocorrendo em áreas úmidas ou periodicamente inundáveis nos campos rupestres (I. Cordeiro, comunicação pessoal), havendo eventuais registros em cerrado (M. F. Calió, comunicação pessoal). No PMM ocorre nas margens dos cursos d'água.

Coleta de dados - Para a investigação sobre as fenofases reprodutivas, 47 indivíduos adultos de $P$. montana foram marcados, entre as coordenadas $12^{\circ} 59^{\prime} 34^{\prime \prime} \mathrm{S}, 41^{\circ} 20^{\prime} 30^{\prime \prime} \mathrm{W}$ e $12^{\circ} 59^{\prime} 28^{\prime \prime} \mathrm{S}, 41^{\circ} 20^{\prime} 19^{\prime \prime} \mathrm{W}$, ao longo das margens do Rio Piabinha e de um pequeno córrego afluente. A quantidade de botões, flores e frutos maduros (em fase de dispersão) de cada indivíduo foram registrados mensalmente, de junho de 2006 a agosto de 2007.

Os dados climatológicos de precipitação acumulada, temperatura e umidade relativa do ar foram coletados em duas estações climatológicas, uma no PMM e outra na Fazenda Agropecuária São João do Paraguaçu, ambos em Mucugê. Os dados mensais de fotoperíodo foram calculados a partir das coordenadas geográficas do PMM, usando o Photoperiod Calculator (Lammi 2005).

Análise dos dados - Os padrões fenológicos de floração foram analisados de acordo com a classificação proposta por Newstrom et al. (1994), baseada em critérios de frequiência e de duração da fenofase de floração, sendo identificadas as categorias de pré-floração (botões), floração (flores abertas) e frutificação (frutos em fase de dispersão).

Para determinar a sincronia de floração, foi registrado o índice de atividade, verificando mensalmente a porcentagem 
de indivíduos floridos (Bencke \& Morellato 2002b). Esse método tem caráter quantitativo em nível populacional e estima a sincronia de floração entre os indivíduos de uma população, sendo que quanto maior o número de indivíduos floridos ao mesmo tempo, maior é a sincronia de floração desta população (Bencke \& Morellato 2002b). A sincronia de floração seguiu a classificação sugerida por Bencke \& Morellato (2002a), sendo reconhecidas as categorias: assincrônica (até $20 \%$ de indivíduos floridos); sincronia baixa (21\%-60\% de indivíduos floridos) e sincronia alta (acima de 60\% de indivíduos floridos).

Para verificar a normalidade da distribuição dos dados utilizou o teste de Shapiro e Wilk (Zar 1996). Como os dados não apresentaram distribuição normal, utilizou-se a correlação de Spearman para relacionar o número de botões, flores e frutos maduros produzidos a cada mês com as variáveis climáticas (San Martin-Gajardo \& Morellato 2003).

\section{Resultados}

A floração de Prepusa montana foi anual e com duração intermediária, ocorrendo entre os meses de junho a setembro de 2006 e abril a julho de 2007, com maior produção de flores em junho (figura 1).

A fenofase de floração coincidiu com o período de menor precipitação média acumulada (figura 1A) e menor umidade relativa do ar (figura 1B), porém, não houve correlação significativa com essas duas variáveis ambientais respectivamente $\left(r_{S}=-0,417553, P>0,05\right.$, $\left.r_{S}=0,176312, P>0,05\right)$.

A floração ocorreu no período de menor comprimento do dia e nos meses mais frios (figura $1 \mathrm{C} \mathrm{e} \mathrm{D),} \mathrm{apresentando}$ correlação negativa significativa com o fotoperíodo $\left(r_{S}=\right.$ $-0,874188, P<0,05)$ e com a temperatura $\left(r_{S}=-0,706379\right.$, $P<0,05)$. A pré-floração também apresentou correlação negativa significativa com o fotoperíodo $\left(r_{S}=-0,682383\right.$, $P<0,05)$.

A sincronia de floração, em 2006, foi alta em junho e julho e baixa em agosto. Em 2007, em abril, a floração foi assincrônica, havendo baixa sincronia nos meses de maio e julho e alta em junho (tabela 1).

A maturação dos frutos e dispersão das sementes iniciaram na estação seca, com o maior número de frutos maduros no início da estação chuvosa (figura 1A), contudo, não houve correlação significativa com a precipitação média acumulada $\left(r_{S}=-0,323715, P>0,05\right)$.

A frutificação ocorreu no período de menor umidade relativa do ar (figura 1B), apresentando uma correlação negativa significativa com esta variável ambiental $\left(r_{S}=\right.$ $-0,546571, P<0,05)$; a abertura dos frutos ocorreu no período com menor comprimento do dia e com temperatura médias mais baixas (figura $1 \mathrm{C}$ e D), não apresentando
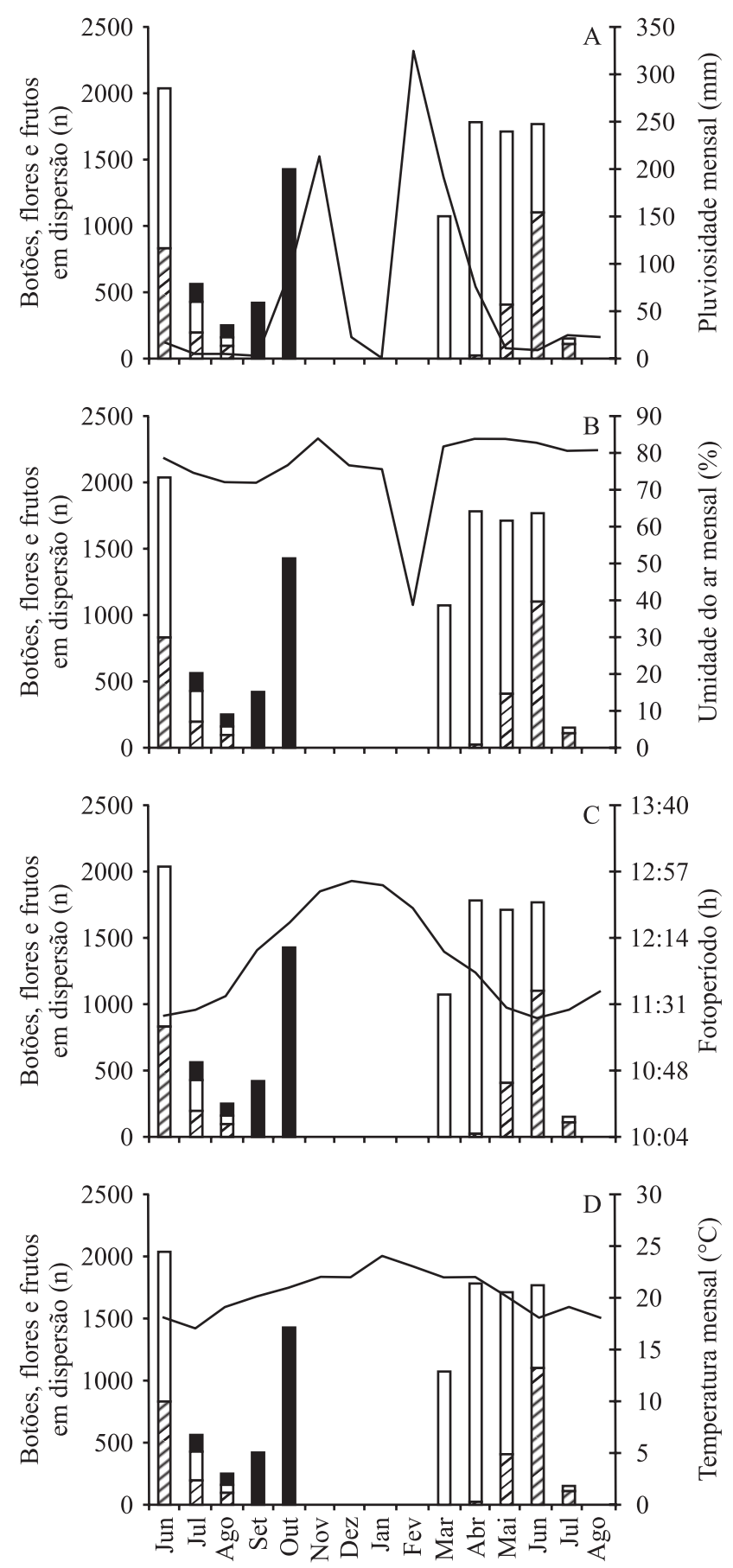

Figura 1. Número total de flores ( $\square$ ), botões $(\square)$ e frutos em dispersão ( $\boldsymbol{\square}$ ) de $P$. montana em uma área de campo rupestre na Chapada Diamantina e dados climáticos mensais no período de junho de 2006 a agosto de 2007. A linha contínua representa em $\mathrm{A}=$ pluviosidade, $\mathrm{B}=$ umidade do ar, $\mathrm{C}=$ fotoperíodo e $\mathrm{D}=$ temperatura.

Figure 1. Total number of flowers ( $\square$ ), buds ( $\square$ ) and mature fruits ( $)$ of $P$. montana in an area of campo ruprestre in the Chapada Diamantina and monthly climatic data between $6 / 2006$ and 8/2007. The line shows in $\mathrm{A}=$ rainfall, $\mathrm{B}=$ relative humidity, $\mathrm{C}=$ photoperiod and $\mathrm{D}=$ temperature. 
Tabela 1. Índice de atividade (IA) da fenofase de floração ou porcentagem de indivíduos floridos em 47 indivíduos de P. montana acompanhados de junho de 2006 a agosto de 2007 em área de campo rupestre da Chapada Diamantina. (* Mês com maior número de flores).

Table 1. Activity index (IA) of flowering or percentage of flowering individuals for 47 individuals of $P$. montana observed between 6/2006 and 8/2007 in an area of campo ruprestre in the Chapada Diamantina. (* Month with more flowers).

\begin{tabular}{lc}
\hline Ano/mês & Índice de atividade $(\%)$ \\
\hline 2006 & \\
Junho* & 89,0 \\
Julho & 70,0 \\
Agosto & 42,0 \\
2007 & \\
Abril & 8,5 \\
Maio & 44,0 \\
Junho* & 68,0 \\
Julho & 42,0 \\
\hline
\end{tabular}

correlação $\left(r_{S}=0,013750, P>0,05\right)$ e $\left(r_{S}=-0,274374\right.$, $P>0,05)$.

As sementes de P. montana permanecem no fruto aberto por até dois meses sendo dispersadas gradualmente. Após liberação de todas as sementes, o ramo fenece, ocorrendo a formação de novos ramos, os quais darão início a um novo ciclo reprodutivo.

\section{Discussão}

A fenologia reprodutiva de Prepusa montana está relacionada com a distribuição sazonal da chuva, apresar de não haver uma correlação significativa entre a pluviosidade e a formação de flores e frutos. Ramos (2005), Colaço et al. (2006) e Costa et al. (2007) também registraram, nas espécies de plantas de campo rupestre que estudaram na Chapada Diamantina, uma maior produção de flores na estação seca; Machado et al. (2007) observaram, na mesma área do presente estudo, que cerca de $50 \%$ da comunidade de plantas visitadas por beija-flores, floresce neste período.

Estes registros, realizados em espécies de plantas zoófilas, sugerem que há uma vantagem adaptativa quanto ao florescimento nos meses mais secos, uma vez que há, neste período, a disponibilização de recursos alimentares importantes para a fauna residente, que utiliza recursos florais, garantindo para estas plantas que suas flores sejam procuradas e visitadas com conseqüente aumento de seu sucesso reprodutivo, através da polinização cruzada (Machado et al. 2007).

A ação das chuvas nas anteras deiscentes, como já foi registrada em Gentiana algida Pallas (Bynum \& Smith 2001), é um fator que provoca diminuição no sucesso reprodutivo, causando danos e remoção do pólen, afetando a viabilidade polínica (Dafni 1992). Portanto, a floração em época de menor pluviosidade pode ser uma importante adaptação de $P$. montana, maximizando seu sucesso reprodutivo.

Por ocorrer em margens de cursos d'água, a floração de $P$. montana não é influenciada pelo estresse hídrico causado pela baixa precipitação na estação seca. A disponibilidade de água para plantas, em ambiente ripário, pode estar desvinculada da pluviosidade (Rodrigues \& Shepherd 2000), uma vez que o solo funciona como um reservatório (Williams-Linera \& Meave 2002), disponibilizando, em época de estiagem, a água necessária para a formação de flores e frutos. Miranda (2007) também observou que espécies em matas ciliares, na Chapada Diamantina, também tem seus padrões fenológicos independentes do regime de chuvas, uma vez que há água disponível no solo ao longo do ano todo.

A floração de $P$. montana está diretamente relacionada aos dias mais curtos e frios. Segundo Rivera \& Borchert (2000), o declínio do fotoperíodo induz o início da floração de espécies em florestas secas. Além disso, a correlação entre o desenvolvimento dos botões florais e o fotoperíodo (Rivera \& Borchert 2000), meses antes do período de abertura floral, foi essencial para haver tempo necessário da indução da antese floral de P. montana nos dias mais curtos e de menores temperaturas. A influência da baixa temperatura na abertura floral em Gentianaceae foi também registrada por Ya-P et al. (2006) em Gentiana straminea Maxim.

Segundo Gentry (1974), o padrão de floração que $P$. montana apresenta é do tipo "cornucopia", caracterizado pela produção numerosa de flores, durante poucas semanas, atraindo muitas espécies de visitantes florais oportunistas. Portanto, a floração de P. montana, em época seca, oferece recurso abundante, atraindo grande diversidade de polinizadores potenciais, como observado em campo. Além disso, a sincronia de floração de $P$. montana, principalmente no pico de floração, pode promover o cruzamento entre indivíduos distantes, proporcionando a diversidade genética e uma menor competição intra-específica (Rathcke \& Lacey 1985).

Assim como observaram Morellato \& Leitão-Filho (1992), em uma comunidade de mata mesófila, e Ramos (2005), em espécies de Eriocaulaceae, também em campo 
rupestre na Chapada Diamantina, o pico de dispersão das sementes de $P$. montana no início da estação chuvosa pode estar relacionado com as condições favoráveis para a germinação e estabelecimento das plântulas no período de maior disponibilidade hídrica superficial, uma vez que as plântulas ainda não possuem sistema radicular desenvolvido o bastante para utilizar reservas menos superficiais de água no solo, provenientes dos reservatórios de água formados em ambiente ripário.

A correlação negativa entre a umidade e a abertura dos frutos, ainda no período seco, é importante para o processo de dessecação das cápsulas e é necessária para a maturação e dispersão inicial dos diásporos, como relatado por Janzen (1967), em espécies de Leguminosae, Bombacaceae, Asclepiadaceae e Bignoniaceae, e por Ramos (2005), em eriocauláceas.

Os frutos de $P$. montana têm o vento como agente dispersor de suas sementes no período inicial de sua maturação, uma vez que a morfologia destas tem atributos anemocóricos (van der Pijl 1982). As chuvas do início da estação chuvosa, quando ocorre o período de pico de dispersão dos diásporos, provoca a retirada das sementes dos frutos deiscentes de P. montana, as quais são levadas até os cursos d'água, onde são carregadas até outros pontos das margens, onde podem encontrar o micro ambiente adequado para seu estabelecimento.

Segundo van der Pijl (1982) existe uma grande dificuldade em descrever a síndrome da dispersão de sementes pela água (hidrocoria). Entretanto, sabe-se que esse fenômeno é geralmente relacionado com a anemocoria, uma vez que ambas são influenciadas por fatores abióticos (van der Pijl 1982). Uma forte relação da chuva na dispersão de frutos deiscentes já foi sugerida por Griz \& Machado (2001), em área de caatinga. Assim, uma investigação mais detalhada sobre a estrutura da semente de $P$. montana pode esclarecer quais os tipos de agentes dispersores são mais eficientes. Sugere-se, então, que o padrão de frutificação de $P$. montana iniciado na estação seca e tendo seu pico no início da estação chuvosa utiliza tanto o vento quanto a água das chuvas e dos cursos d'água como agentes dispersores de suas sementes.

Agradecimentos - Agradecemos à Prefeitura Municipal de Mucugê, BA, pelo apoio logístico e a seus funcionários pela ajuda durante os trabalhos de campo; a C.O.C. Ramos e R.B. Fonseca pelas importantes sugestões; à M.F. Calió, M. Cassaro e a Dra. I. Cordeiro, pela atenção e preciosas informações; a C.S. Santana pela valiosa ajuda em campo e às biólogas e estagiários do Lorma/UEFS pelo auxilio e companhia em laboratório; agradecemos também a Capes pelo suporte financeiro - Capes Demanda Social: 68759641 e ao Programa de Pós-graduação em Botânica/UEFS.

\section{Referências Bibliográficas}

ALBERT, V.A. \& STRUWE, L. 2002. Gentianaceae in context. In Gentianaceae: systematics and natural history (L. Struwe \& V.A. Albert, eds.). Cambridge University Press, Cambridge p.1-20.

BENCKE, C.S.C. \& MORELLATO, L.P.C. 2002a. Estudo comparativo da fenologia de nove espécies arbóreas em três tipos de floresta atlântica no sudeste do Brasil. Revista Brasileira de Botânica 25:237-248.

BENCKE, C.S.C. \& MORELLATO, L.P.C. 2002b. Comparação de dois métodos de avaliação da fenologia de plantas, sua interpretação e representação. Revista Brasileira de Botânica 25:269-275.

BYNUM, M.R. \& SMITH, W.K. 2001. Floral movements in response to thunderstorms improve reproductive effort in the alpine species Gentiana algida (Gentianaceae). American Journal of Botany 88:1088-1095.

COLAÇO, M.A.S., FONSECA, R.B.S., LAMBERT, S.M, COSTA, C.B.N., SANTOS, C.G.M. \& BORBA, E.L. 2006. Biologia reprodutiva de Melocactus glaucescens Buining \& Brederoo e Melocactus paucispinus G. Heimen \& R. Paul (Cactaceae), na Chapada Diamantina, Nordeste do Brasil. Revista Brasileira de Botânica 29: 239-249.

CONCEIÇÃO, A.A. \& GIULIETTI, A.M. 2002. Composição florística e aspectos estruturais de campo rupestre em dois platôs do Morro do Pai Inácio, Chapada Diamantina, Bahia, Brasil. Hoehnea 29:37-48.

CONCEIÇÃO, A.A. \& PIRANI, J.R. 2005. Delimitação de habitats em campos rupestres na Chapada Diamantina, Bahia: substratos, composição florística e aspectos estruturais. Boletim de Botânica da Universidade de São Paulo 23:85-111.

COSTA, C.B.N., LAMBERT, S.M., BORBA, E.L. \& QUEIROZ, L.P. 2007. Post-zygotic reproductive isolation between sympatric taxa in the Chamaecrista desvauxii complex (Leguminosae-Caesalpinioideae). Annals of Botany 99:625-635.

DAFNI, A. 1992. Pollination ecology: a practical approach. University Press, New York, Oxford.

FAEGRI, K. \& VAN DER PIJL, V.D. 1979. The principles of pollination ecology. $3^{\text {th }}$ ed. Pergamon Press, New York.

GENTRY, H.A. 1974. Flowering phenology and diversity in tropical Bignoniaceae. Biotropica 6:64-68.

GIULIETTI, A.M., PIRANI, J.R. \& HARLEY, R.M. 1997. Espinhaço Range Region, Eastern Brazil. In Centres of Plant Diversity: a guide and strategy for their conservation. (S.D. Davis, V.H. Heywood, O. HerreraMacBryde, J. Villa-Lobos \& A.C. Hamilton, eds.). Information Press, Oxford 3:397-404.

GRIZ, L.M.S. \& MACHADO, I.C. 2001. Fruiting phenology and seed dispersal syndromes in caatinga, a tropical dry forest in the northeast Brazil. Journal of Tropical Ecology 17:303-321. 
HARLEY, R.M. 1995. Introduction. In Flora of the Pico das Almas, Chapada Diamantina - Bahia, Brasil (B.L. Stannard, ed.). The Trustees of the Royal Botanic Gardens, Kew, p.1-42.

JANZEN, D.H. 1967. Synchronization of sexual reproduction of trees within the dry season in Central America. Evolution 21:620-37.

LAMMI, J. 2005. On line photoperiod calculator. http:// www.etti.fi/ jjlammi/sun.htm1 (acesso em 01/09/2007).

LENNARTSSON, T. 1997. Seasonal differentiation - a conservative reproductive barrier in two grassland Gentianella (Gentianaceae) species. Plant Systematics and Evolution 208:45-69.

LENNARTSSON, T., OOSTERMEIJER, J.G.B., VAN DIJK, J. \& DEN NIJS, H.C.M. 2000. Ecological significance and heritability of floral reproductive traits in Gentianella campestris (Gentianaceae). Basic and Applied Ecology 1:69-81.

MACHADO, C.G., COELHO, A.G., SANTANA, C.S., RODRIGUES, M. 2007. Beija-flores e seus recursos florais em uma área de campo rupestre da Chapada Diamantina, Bahia. Ararajuba, Revista Brasileira de Ornitologia 15:215-227.

MIRANDA, L.D.P. 2007. Fenologia vegetativa de espécies arbóreas de mata ciliar e mata de encosta, Lençóis, Chapada Diamantina, Bahia. Tese de doutorado, Universidade Estadual de Feira de Santana, Feira de Santana.

MORELLATO, L.P.C. \& LEITÃO-FILHO, H.F. 1992. Padrões de frutificação e dispersão na Serra do Japi. In História natural da Serra do Japi: ecologia e presevação de uma área florestal no Sudeste do Brasil (L.P.C. Morellato, org.). Editora da Unicamp/Fapesp, Campinas, p.112-140.

NEWSTROM, L.E., FRANKIE, G.W. \& BAKER, H.G. 1994. A new classification for plant phenology based on flowering patterns in lowland tropical rain forest trees at La Selva, Costa Rica. Biotropica 26:141-159.

PETANIDOU, T., ELLIS-ADAM, A.C., DEN NIJS, J.C.M, \& OOSTERMEIJER, J.G.B. 1998. Pollination ecology of Gentianella uliginosa, a rare annual of the Dutch coastal dunes. Nordic Journal of Botany 18:537-548.

PETANIDOU, T., ELLIS-ADAM, A.C., DEN NIJS, H.C.M. \& OOSTERMEIJER, J.G.B. 2001. Differential pollination success in the course of individual flower development and flowering time in Gentiana pneumonanthe L. (Gentianaceae). Botanical Journal of the Linnean Society 135:25-33.

RAMOS, C.O.C. 2005. Fenologia e biologia reprodutiva de Syngonanthus mucugensis Giul e S. curralensis Moldenke (Eriocaulaceae), nos municípios de Mucugê e Morro do Chapéu, Chapada Diamantina, Bahia, Brasil. Dissertação de mestrado, Universidade Estadual de Feira de Santana, Feira de Santana.
RATHCKE, B. \& LACEY, E.P. 1985. Phenological patterns of terrestrial plants. Annual Review of Ecology and Systematics 16:179-214.

RIVERA, G. \& BORCHERT, R. 2000. Induction of flowering in tropical trees by a 30-min reduction in photoperiod: evidence from field observations and herbarium collections. Tree Physiology 21:201-212.

RODRIGUES, R.R. \& SHEPHERD, G.J. 2000. Fatores condicionantes da vegetação ciliar. In Matas ciliares: conservação e recuperação (R.R. Rodrigues \& H.F. Leitão-Filho, eds.) Edusp/Fapesp, São Paulo, p.101-107.

SAN MARTIN-GAJARDO, I. \& MORELLATO, L.P.C. 2003. Fenologia de Rubiaceae do sub-bosque em floresta Atlântica no sudeste do Brasil. Revista Brasileira de Botânica 26:299-309.

SPIRA, T.P. \& POLLAK, O.D. 1986. Comparative reproductive biology of alpine biennial and perennial gentians (Gentiana: Gentianaceae) in California. American Journal of Botany 73:39-47.

STRADMAN, M.T.S. 1998. Plano de Manejo do Parque Municipal de Mucugê. Projeto Sempre Viva. Prefeitura Municipal de Mucugê, Mucugê.

STRUWE, L., KADEREIT, J.W., KLACKENBERG, J., NILSSON, S., THIV, M., VON HAGEN, K.B., \& ALBERT, V.A. 2002. Systematics, character evolution, and biogeography of Gentianaceae including a new tribal and subtribal classification. In Gentianaceae: systematics and natural history (L. Struwe \& V.A. Albert, eds.). Cambridge University Press, Cambridge, p.210-309.

VAN DER PIJL, L. 1982. Principles of dispersal in higher plants. $3^{\text {th }}$ ed. Springer-Verlag, Berlin.

VILLARREAL, J.A.Q. 1998. Flora del Bajio e de regiones adyacentes: Familia Gentianaceae. Fasciculo 65. Instituto de Ecología A.C. Pátzcuaro, Michoacán.

WAGNER, J.E. MITTERHOFER, R. 1998. Phenology, seed development, and reproductive success of an alpine population of Gentianella germanica in climatically varying years. Botanica Acta 111:159-166.

WILLIAMS-LINERA, G. \& MEAVE J.A. 2002. Patrones fenológicos de bosque lluvioso neotropical de bajura. In Ecología de bosques lluviosos Neotropicales (M. Guariguata \& G. Kattan, eds.). IICA, San Jose, p.407-431.

WOLFF, D.A. 2005. Pollination Biology of Gentianales in a Southern Ecuadorian Montane Forest. Tese de doutorado, Fakultät für Bilogie, Chemie und Geowissenschaften der Universität Bayreuth. Bayreuth.

YA-P, H., YUAN-W, D., JIAN-Q, L. \& SMITH, W.K. 2006. Floral closure in response to temperature and pollination in Gentiana straminea Maxim. (Gentianaceae), an alpine perennial in the Qinghai-Tibetan Plateau. Plant Systematics and Evolution 256:17-33.

ZAR, J.H. 1996. Bioestatistical analysis. Prentice-Hall, New Jersey. 